\title{
Thermal Load and Heat Transfer in Dental Titanium Implants: an Exact Analytical Solution to the 'Heat Equation'
}

\author{
Ziyad S. Haidar ${ }^{1-4 *}$ \\ ${ }^{1}$ BioMAT'X, Universidad de los Andes, Santiago, Chile. \\ ${ }^{2} \mathrm{CiiB}$, Universidad de los Andes, Santiago, Chile. \\ ${ }^{3}$ Programa de Doctorado en BioMedicina, Facultad de Medicina, Universidad de los Andes, Santiago, \\ Chile. \\ ${ }^{4}$ Facultad de Odontología, Universidad de los Andes, Santiago, Chile.
}

\section{CORRESPONDENCE:}

Ziyad S. Haidar. DDS, Cert Implantol, MSc OMFS, FRCS(C), MBA, PhD. Professor and Scientific Director, Faculty of Dentistry, Universidad de Los Andes, Santiago de Chile. Founder and Head of BioMAT'X (Laboratorio de Biomateriales, Farmacéuticos y Bioingeniería de Tejidos Cráneo Máxilo-Facial), Centro de Investigación e Innovación Biomédica (CiiB), I+D+i Department, Universidad de Los Andes, Mons. Álvaro del Portillo 12.455 - Las Condes, Santiago de Chile. Telephone: +56 2 26181372; Fax: +56 2 22149468; E-mail address: zhaidar@uandes.cl

\begin{abstract}
Introduction: Heat is a kinetic process whereby energy flows from between two systems; hotto-cold objects. In oro-dental implantology, conductive heat transfer/(or thermal stress) is a complex physical phenomenon to analyze and consider in treatment planning. Hence, ample research has attempted to measure heat-production to avoid over-heating during bone-cutting and -drilling for titanium (Ti) implant-site preparation and insertion, thereby preventing/minimizing early (as well as delayed) implant-related complications and failure. Objective: Given the low bone-thermal conductivity whereby heat generated by osteotomies is not effectively dissipated and tends to remain within the surrounding tissue (peri-implant), increasing the possibility of thermal-injury; this work attempts to obtain an exact analytical solution of the heat equation under exponential thermal-stress, modeling transient heat transfer and temperature changes in $\mathrm{Ti}$ implants upon hot-liquid intake. Materials and Methods: We investigate the impact of the material, the location point along implant length, and the exposure time of the thermal load on temperature changes. Results: Despite its simplicity, the presented solution contains all the physics and reproduces the key features obtained in previous numerical analyses studies. To the best of knowledge, this is the first introduction of the intrinsic time, a "proper" time that characterizes the geometry of the dental implant, where we show, mathematically and graphically, how the interplay between "proper" time and exposure time influences temperature changes in Ti implants, under the suitable initial and boundary conditions. Conclusions: This work aspires to accurately complement the overall clinical diagnostic and treatment plan for enhanced bone-implant interface, implant stability and success rates, whether for immediate or delayed loading strategies.
\end{abstract}

Keywords: Dental implants; Thermal stress; Modeling of heat transfer; Temperature changes; Heat equation; Analytical solution. 


\section{INTRODUCTION}

Despite significant progress in the diagnosis, prevention, management and treatment of orodental diseases, teeth and supporting tissues either damaged or lost due to disease or trauma, continue to embody a burden. The quality of life of men and women of all ages Worldwide is affected by a missing tooth in several ways. Speaking difficulties, pain, loss of confidence and poor eating (chewing/mastication) capacity, are only a few. Thus, reduction in number of teeth may deteriorate quality of life [1]. Also, several previously reported articles stated that missing teeth are closely related to death $[2,3,4]$. Nowadays a number of options exist for the replacement of missing teeth. Dental implants are used to replace missing teeth [5], a great option for patients missing natural teeth, because they act as a secure anchor for artificial replacement teeth and eliminate the instability associated with surface adhesives and removable bridges [6]. Hence, dental implants, titanium-based mainly, are a predictable treatment modality for the rehabilitation of partially and completely edentulous patients. Indeed, dental implant use has nearly tripled since 1986 [6a], and it is expected to continue to rise or grow, rapidly. People of all ages are turning to dental implants to replace a single tooth, several teeth or a full set of dentures. Leading reasons for choosing dental implants are: to restore normal eating and speaking abilities, to enhance facial appearance and confidence, and to increase denture retention. Dental implants changed (and continue to) the way people live, they are re-discovering the comfort and confidence to eat, speak, laugh and enjoy life [6].

Due to consumption of hot foods and liquids, the human tooth is daily subjected to thermal loading. Heat generated on the tooth surface from intra-oral temperature changes is transferred via conduction through the enamel, dentin and pulp. Since enamel and dentin have lower values of thermal conductivity, the pulp is protected against rapid thermal fluctuations [7]. The thermal behavior, however, of restored teeth is significantly different in comparison to intact teeth, as the metals used in clinical restorative applications, such as titanium or titanium alloy, are excellent thermal conductors $[8,9,10,11,12,13]$. High temperatures may cause irreversible damage to tissues and organs [14], while the habitual consumption of extremely hot foods and beverages may affect implant treatment modality. Mechanical stability of dental implants is a prerequisite for successful rehabilitative and restorative therapy, and furthermore, it can be stated that the cornerstone of successful dental implant therapy is an intact biological osseointegration around the implant (fixture), thereby playing an important role in provision of the pursued stability. Osteoblast cells require in situ activation to increase bone density and establish high anchorage and subsequent high stability, survival and success of the implant [15]. Thermal injury to the implant-bone interface may lead to bone necrosis and loss of osseointegration. Previous studies have shown that osteoblasts may be severely damaged by a thermal impulse of 42 degrees ( 10 minute heat shock) [16], and that some bone proteins are lost [17]. Furthermore, it was stated that the temperature threshold for necrosis of the bone (cortical) is 47 degrees (for 1 minute) [18, 19, 20]. Yet, literature reports intra-oral temperatures reaching 67-77 degrees during the consumption of hot water $[21,22]$.

Intra-bony heat generation, during surgical implant insertion, is another story (alarming), with few serious reports on temperatures at the implant-bone interface, whether during, postsurgical preparation and/or during and post-hot substance consumption. Questions pertinent to threshold level(s) and probable transient changes in osteoblasts are raised.

Thus, the transient heat transfer under thermal load is of vital significance in dentistry, in general, and in practical oro-dental implantology, in specific. In the literature already exist some previous works on the subject, where the authors attempt to model and investigate the effects of "thermal load(s)" on the bone-implant interface system [23, 24, 25, 26, 27] (see, 
however, e.g. [27a,27b] for heat transfer from warm water to foot in a footbath). In this work, our approach is distinct in two main respects: (1) in the other studies, authors tend to solve the heat equation with a source term that accounts for the temperature excess T-Ts, with (T) being the temperature at a given point and (Ts) the temperature from the surroundings. Herein, we find it more natural to integrate the heat equation without the additional source term and take into account the thermal load into the imposed boundary conditions; and (2) in other works, the investigation is often based on numerical analysis, and, to the best of our knowledge, an exact analytical solution, is still missing. Obtaining an exact analytic expression for the solution is always challenging and desirable, since the physics is more transparent, while at the same time is is accessible to everyone. In particular, the interested reader may use the analytic expression either to check/reproduce the results shown in articles or to study other aspects of the solution not considered in publications.

Therefore, in the present work, our goal is two-fold: (A) We propose to solve the standard heat equation, modifying the imposed boundary conditions without any additional source term, and on the other hand (B) We fill a gap in the literature by obtaining an exact analytical solution of a somewhat simplified problem, which nevertheless, encapsulates the physics and reproduces the results already found in previous works via numerical analyses. In addition, for the first time, we introduce and involve herein, the intrinsic time, a "proper" time that characterizes the geometry of the dental implant fixture and overall system, and we show how the interplay between that time and the exposure time influences temperature changes, and subsequent implant survival. Therefore, this work aims to complement the overall clinical diagnostic and treatment plan for enhanced biological bone-implant interface and mechanical implant stability and success rates, whether for immediate or delayed implant loading strategies.

To simplify flow, the plan of our work is the narratively, as follows: Following problem formulation, we obtain an analytical solution, followed with the appropriate figures demonstrating its main features. Two appendices are included for self-completeness and to not interrupt the flow of the discussion. 


\section{THERMAL LOAD AND HEAT TRANSFER}

\section{A. Formulation of the physical problem}

The dental implant system, typically, consists of three parts, namely the root or fixture (good conductor), the abutment (good conductor) as well as the crown (moderate to poor conductor), see Figure 1. In principle, one must solve the full three-dimensional heat equation for the temperature $T(x, y, z)$

$\frac{\partial T}{\partial t}=\alpha\left(\frac{\partial^{2} T}{\partial x^{2}}+\frac{\partial^{2} T}{\partial y^{2}}+\frac{\partial^{2} T}{\partial z^{2}}\right)$

where $\alpha$ is the thermal diffusivity, which is assumed to be a constant, space and temperature independent. For a derivation of the heat equation the interested reader may consult Appendix A. An additional source term $q(t)=m(T-T s)$, where $m$ is a constant depending on the type and the geometry of the implant, may be added to take into account the temperature excess due to the thermal loading $[24,25]$.

Here, however, as already mentioned in the introduction, we propose to solve the standard heat equation (1) and consider thermal stress via imposing the appropriate boundary condition, see the discussion below.

Given the geometry of the implant, it may be modeled as a cylinder with length $L$ and radius $R$, and so one expects to obtain an axisymmetric solution where the temperature will not depend on the rotation angle, and therefore the problem is essentially two-dimensional. During heat transfer along the axis of the implant, heat loss occurs in the radial direction as well. In the present work, however, since we are mostly interested in temperature changes along the axis of the implant, we shall ignore the radial dependence, and thus we shall solve the onedimensional heat equation (an approximation also justified by the fact that typically the length is considerably larger than the radius of the implant) for the temperature $T(t, y)$

$$
\frac{\partial T}{\partial t}=\alpha \frac{\partial^{2} T}{\partial y^{2}}
$$

in the domain $\mathrm{t}>0$, and $0<\mathrm{y}<\mathrm{L}$, assuming for simplicity a single value of the thermal diffusivity throughout the length of the implant, corresponding to that of the abutment.

In this work we shall consider two numerical values between the diffusivity of titanium and that of ceramic. The endpoint $y=0$ corresponds to the bone, while the other endpoint $y=L$ corresponds to the oral cavity.

This partial differential equation must be supplemented with the initial condition $T(t=0, y)=$ $f(y)$ as well as with the two boundary conditions at the surface (oral cavity) and at the bone, namely $T(t, y=L)=T$ surface $(t)$ and $T(t, y=0)=$ Tbone $(t)$, where the functions Tbone $(t)$, Tsurface $(t)$ an $f(y)$ are given functions depending on the physics of the problem at hand. This 
problem is well posed, and it has a unique solution [28]. For example, in the simplest case, in which

$$
\begin{aligned}
& T(t=0, y)=T_{0} \\
& T(t, y=L)=T_{0} \\
& T(t, y=0)=T_{0}
\end{aligned}
$$

where T0 is a constant temperature (e.g. room temperature or body's natural temperature) the unique solution must be $T(t, y)=T 0$, as it clearly satisfies the heat equation and all conditions.

In the present work we are interested in studying the effect of thermal stress, where it is assumed that the temperature of the oral cavity starts from, a high temperature $\mathrm{T} 1=60$ degrees, and then it decreases monotonically until it eventually reaches the body temperature $\mathrm{T} 2=37$ degrees.

We can model this behavior in a simple and at the same time realistic way introducing an elementary function exhibiting a smooth and continuous transition from T1 down to T2. In particular, in the discussion to follow we shall consider an exponential function as follows

$$
T_{\text {oralcavity }}(t)=\left(T_{1}-T_{2}\right) \exp \left(-t / t_{0}\right)+T_{2}
$$

where to is the thermal stress exposure time that shows how fast the temperature of the oral cavity drops to the body's natural temperature (Figure 3). As a matter of fact, if we take t0 $=0.2 \mathrm{sec}$, which corresponds to a duration of approximately $1 \mathrm{sec}$, or t0=2 sec, which corresponds to a duration of $10 \mathrm{sec}$, our thermal loading resembles the ones commonly considered in previous works [21,24,26-30]. Therefore, in what follows we assume an initial condition $T(t=0, y)=T 2$, a boundary condition at the bone $T(t, y=0)=T 2$ and a boundary condition at the surface $T(t, y=L)=$ Toralcavity $(t)$ given in (8), and we define $\Delta T=T 1-T 2=23$ degrees.

\section{B. Exact analytical solution of the initial/boundary value problem}

We now proceed to find the solution of the one-dimensional heat equation with the above initial and boundary conditions. Since the boundary conditions are non-homogeneous, we employ the standard trick by writing $T(t, y)$ as a sum of two functions

$$
T(t, y)=u(t, y)+w(t, y)
$$

where $w(t, y)$ is a particular function that absorbs the non-homogeneous boundary conditions, so that the second function $u(t, y)$ satisfies homogeneous boundary conditions. It is easy to check that the function $w(t, y)$ is given by 


$$
w(t, y)=T_{2}+\frac{\Delta T}{L} y \exp \left(-t / t_{0}\right)
$$

while $u(t, y)$ satisfies the following partial differential equation

$$
\frac{\partial u}{\partial t}-\alpha \frac{\partial^{2} u}{\partial y^{2}}=\frac{\Delta T}{L t_{0}} y \exp \left(-t / t_{0}\right)
$$

and the conditions

$$
\begin{gathered}
u(t=0, y)=-y \frac{\Delta T}{L} \\
u(t, y=L)=0 \\
u(t, y=0)=0
\end{gathered}
$$

We see that the price to pay is that $u(t, y)$ satisfies a non-homogeneous differential equation. However, this does not pose a problem since we can make the following ansatz by expanding on a complete basis of functions

$$
u(t, y)=\sum_{n=1}^{\infty} C_{n}(t) \sin \left(\frac{n y}{L}\right)
$$

where $n=1,2, \ldots$ is an integer, and $C n(t)$ are unknown functions of time depending on $n$. The homogeneous boundary conditions at $y=0, L$ are automatically satisfied, while the coefficients $\mathrm{Cn}(\mathrm{t})$ can now be determined by using the differential equation and the initial condition. First of all we use the fact that any function $g(y)$ can be expanded on the basis $\sin (n \pi y / L)$ in the following form

$$
g(y)=\sum_{n=1}^{\infty} A_{n} \sin \left(\frac{n \pi y}{L}\right)
$$

with coefficients $A_{n}$ that are given by

$$
A_{n}=\frac{2}{L} \int_{0}^{L} d y g(y) \sin \left(\frac{n \pi y}{L}\right)
$$

using the orthogonality of the basis

$$
\int_{0}^{L} d y \sin \left(\frac{n \pi y}{L}\right) \sin \left(\frac{m \pi y}{L}\right)=\frac{L}{2} \delta_{n, m}
$$

where $\delta n, m$ is the Kronecker symbol taking the value 1 when $n=m$ and 0 otherwise. For the function $g(y)=y$ the coefficients are given by 


$$
A_{n}=\frac{(-1)^{n+1} 2 L}{\pi n}
$$

Now the initial condition implies $C n(0)=-\Delta T$ An/L, while if we plug the ansatz into the partial differential equation we obtain an ordinary differential equation for $\mathrm{Cn}(\mathrm{t})$ as follows

$$
\frac{d C_{n}(t)}{d t}+\alpha\left(\frac{n \pi}{L}\right)^{2} C_{n}(t)=\frac{\Delta T A_{n}}{L t_{0}} \exp \left(-t / t_{0}\right)
$$

In Appendix B, we describe how to obtain the solution to the above differential equation. Putting everything together, and defining $\tau=L^{2} /\left(\alpha \pi^{2}\right)$ to be the intrinsic time of the dental implant, the exact analytical solution to the initial/boundary value problem is given by

$$
\begin{aligned}
& T(t, y)=T_{2}+\frac{\Delta T}{L} y \exp \left(-t / t_{0}\right)+\frac{2 \Delta T}{\pi} \sum_{n=1}^{\infty} D_{n}(t) \sin \left(\frac{n \pi y}{L}\right) \\
& D_{n}(t)=\frac{(-1)^{n+1}}{n}\left[\frac{\exp \left(-t / t_{0}\right)}{-1+n^{2}\left(t_{0} / \tau\right)}-\left(1+\frac{1}{-1+n^{2}\left(t_{0} / \tau\right)}\right) \exp \left(-n^{2} t / \tau\right)\right]
\end{aligned}
$$

We notice that the solution does not depend on $L$ and $\alpha$ separately, but only through the intrinsic time $\tau$. It is easy to check that the initial and boundary conditions are satisfied. Also we see that the above solution approaches the body's natural temperature after sufficiently long time, $T(t, y) \rightarrow T 2$ as $t \rightarrow \infty$.

This is to be expected, since the temperature of the oral cavity eventually drops to the body's natural temperature, and from that moment on the unique solution of the heat equation with constant initial/boundary conditions corresponds to the constant temperature $T(t, y)=T 2$. As we will see shortly, the temperature changes versus time at a certain location, e.g. close to the surface or close to the bone, depend on the interplay between the exposure time to and the intrinsic time of the implant $\tau=L^{2} /\left(\alpha \pi^{2}\right)$, which for a given length $L$ is low for good thermal conductors and high for poor conductors. We remark in passing that in works studying thermal therapies, a very important concept is that of thermal dose. First described by Sapareto and Dewey [30a], it is computed cumulatively using an empirical formula, and in practice the way it works is that for every degree above 43 the required time to coagulate the tissue halves, i.e. 120 minutes at 44, 60 minutes at 45 etc. For more details see also [30b].

For the length considered here ( $L=1.3 \mathrm{~cm}$, see next section), for titanium the intrinsic time is found to be $\tau=1.9 \mathrm{sec}$, while for ceramic it is computed to be $\tau=27.4 \mathrm{sec}$ (Appendix A).

\section{MAIN FEATURES OF THE SOLUTION}

Herein, we attempt to demonstrate, in a pictorial way, the behavior and main features of the exact analytical solution obtained in the previous section. This is accomplished through a series of figures, in which we demonstrate/show temperature changes versus time for i) two types of implants $A$ and $B$; ii) several different numerical values of the exposure time t0; and iii) the 
location point along the axis of the implant. Our approach is theoretical, with no experimental validation feasible or at disposal. Still, the figures below clearly show that all key features observed in the earlier related studies are reproduced.

The system in principle is characterized by three free parameters, namely the length of the implant $L$, the thermal diffusivity of the material $\alpha$, and the exposure time to of the thermal load, although in practice the behavior of the solution depends on the interplay between t0 and the intrinsic time of the implant (see discussion below).

$$
\tau=L^{2} / \alpha
$$

In the following we find it natural to split the free parameters in two ways, as follows: (i) from the one hand the duration of the load, roughly $5 \mathrm{t0}$; and (ii) on the other hand the intrinsic time $\tau$ of the geometry of the dental implant. The length of the dental implant varies from 7 $\mathrm{mm}$ to $20 \mathrm{~mm}$ [23]. Here we fix the length at $L=1.3 \mathrm{~cm}$ as in [24]. Moreover, for a given geometry and a certain thermal load, temperature changes depend on the location along the implant. Following the standard notation, we introduce the location points $B 1$ at $y=3 \mathrm{~L} / 4$ (superficial), B2 at $\mathrm{y}=\mathrm{L} / 2$ (middle) and B3 at $\mathrm{y}=\mathrm{L} / 4$ (deep) [25]. Finally, we consider two different types of implants, type A with $\alpha=2 \times 10^{-6}\left(\mathrm{~m}^{2} / \mathrm{s}\right)$ (moderate conductor), and type $\mathrm{B}$ with $\alpha=5 \times 10^{-6}\left(\mathrm{~m}^{2} / \mathrm{s}\right)$ (good conductor), with values comparable to those employed in $[24,25]$; consequently, our approach and findings may be directly compared to the results obtained therein.

The main features of the obtained analytic solution for the implants $A$ and $B$ as well as for several values of the exposure time is shown graphically in Figures 4-8.

$\alpha$

Recall that for a given thermal load (i.e. known t0) and for given implant material (i.e. known thermal diffusivity ), the temperature depends on two independent variables, namely from the one hand on the time $t$ and on the other hand on the location point $y$ along the axis of the implant. Therefore, one may plot $T$ versus $t$ for a certain point $y$, or plot $T$ versus $y$ at a given instant of time. This is shown in Figures 4 and 5 below.

First, to see how temperature varies along the implant at a given instant of time, in Figure 4 we show the temperature distribution from $y=0$ up to $y=L$ at four different instants of time, $t=9,12,15$ and $18 \mathrm{sec}$, for implant $A$ and for $t 0=2 \mathrm{sec}$. At every instant of time the temperature at the end points remains the same due to the imposed boundary conditions, while at a certain location, i.e. fixed y point, the temperature decreases with time.

The impact on the temperature of the location point along the implant is shown in Figure $\mathbf{5}$, where we show temperature varies with time at points $B 1, B 2$ and $B 3$ for implant $A$ and $t 0=2$ sec. As we go deeper the maximum temperature reached decreases and the time needed to reach it increases. The highest temperature is observed at point B1 due to its proximity to the thermal load.

To see the impact of the material chosen on temperature changes, in Figure 6 we show temperature changes for both implants $A$ and $B$ at point $B 2$ for t $0=2 \mathrm{sec}$. The good conductor (type $B$ in brown) reaches the highest temperature fast, while the moderate conductor (type $A$ in orange) reaches a lower highest temperature later, due to the fact the heat is transferred slower in the case of implant A. Our results shown in Figure 4 and Figure 5 have been also observed in [24]. 
Finally, in Figures $\mathbf{7}$ and Figure 8, we show temperature changes at point B2 for 5 different exposure times t0 for implants $A$ and $B$, respectively. In particular for implant $A$, with an intrinsic time $8.6 \mathrm{sec}$, we have considered to $=2,5,8,11$ and $14 \mathrm{sec}$, from bottom to top, while for implant $B$, with an intrinsic time $3.4 \mathrm{sec}$, we have considered t0=1,2,3,5 and $6 \mathrm{sec}$, from bottom to top. For both implants the curve in the middle corresponds to the case where the exposure time is very close to the intrinsic time of the implant. We see that the highest temperature observed increases with the exposure time both for implant $A$ and implant $B$. Notice that when the exposure time approaches the intrinsic time of the implant, the highest temperature reached is approximately 41 degrees, just below the critical temperature of 42 degrees, irrespectively of the material chosen.

A typical implant system is made of titanium and metal alloys; hence, a continuous thermal conduction pathway (and heat reservoir) is created between the oral cavity and deeper parts of the jaw bone. Herein, heat conduction is mainly mitigated by implant design and diameter (and time). Temperature at the abutment-implant interface is vital. Likewise, are type and amount of metal in implant composition, whereby in situ heat transfer to supporting perimplant tissues can be significantly accelerated. Therefore, to reduce risks of injury or damage, the exposure time should be lower than the intrinsic time, or, if it is higher it must be as close to the "proper" time as possible. Minimize heat generation during implantology procedures and advise patients to restrain from or avoid hot beverages as much as possible until satisfactory clinical stability or even full osseointegration are evident; especially in cases of delayed implant loading / restoration.

Figures 2-7 have been produced employing a Wolfram (wolfram.com) Mathematica file.

\section{CONCLUSIONS}

The cornerstone of successful dental implant therapy is osseointegration. Despite dental implants being a predictable treatment modality for the rehabilitation of partially and completely edentulous patients, high temperatures may cause irreversible damage to tissues and organs, with undesirable outcomes and sequels. In this work, to summarize, we have addressed the interesting problem of transient heat transfer and temperature changes in titanium dental implants upon hot liquid intake. To that end, we have solved the heat equation with appropriate initial and boundary condition assuming an exponential thermal load modeling the consumption of hot beverages. We have obtained an exact analytical solution filling a gap in the literature, since to the best of our knowledge it was something missing. We have investigated what the impact of the material chosen, the location point along the implant, and the exposure time of the thermal load is on temperature changes. Furthermore, we have introduced in this work for the first time the intrinsic time that characterizes the geometry of the dental implant, and we have shown graphically how the interplay between this "intrinsic" time and the exposure time of the thermal load influences temperature changes. We conclude that the exact analytical solution obtained here, despite its simplicity, encapsulates all the physics, and it nicely reproduces the key features previously obtained in other numerical analyses. 
ACKNOWLEDGMENTS: Generous funding and operating grants supported this work by providing to the BioMAT'X Research Group, part of CiiB (Centro de Investigación e Innovación Biomédica), through the Faculty of Dentistry and Department for Research, Development and Innovation, Universidad de los Andes, Santiago de Chile.

FUNDING: Author (G.P.P.) acknowledges the Fundaçao para a Ciencia e Tecnologia (FCT) in Portugal for the financial support provided to CENTRA (Center for Astrophysics and Gravitation), Instituto Superior Técnico, Universidade de Lisboa, through the Grant No. UIDB/00099/2020. The corresponding author (Z.S.H.) acknowledges supplementary operating funding provided from CONICYT-FONDEF Chile under awarded project/grant (national) \#ID16I10366 (2016-2019) and Fondo de Ayuda a la Investigacion FAI-Universidad de los Andes No. INV-IN-2015-101 (2015-2020).

CONFLICTS OF INTEREST: None. 


\section{APPENDIX A:}

The heat equation can be derived using energy conservation, Fourier's law for the heat flux, $\mathbf{q}$ $=-\mathrm{k} \nabla \mathrm{T}$, with $\mathrm{k}$ being the thermal conductivity of the material, and the expression for heat, $\mathrm{Q}=$ $\mathrm{m} \mathrm{c} \mathrm{T}$, where $\mathrm{c}$ is the specific heat of the material. In the one-dimensional case, applying energy conservation in an infinitesimal volume $A \Delta x$ with mass $\rho A \Delta x$, where $\rho$ is the mass density, we can see that during an infinitesimal time interval $\Delta t$ the heat inside the volume changes according to Fourier's law

$$
c \rho \frac{\Delta T}{\Delta t}=k^{\frac{\partial T}{\partial x}(x+\Delta x)-\frac{\partial T}{\partial x}(x)}
$$

and taking the limit $\Delta x \rightarrow 0$ we finally obtain the heat equation

$$
\frac{\partial T}{\partial t}=\alpha \frac{\partial^{2} T}{\partial x^{2}}
$$

where the thermal diffusivity is given by $\alpha=k /(c \rho)$. For a given material one can find in tables the values of $k, c$, and $\rho$, and then using the previous simple formula he can compute $\alpha$ for that material. Typically, in the International System of units (S.I.) the mass density is of the order of $1000\left(\mathrm{~kg} \mathrm{~m}^{-3}\right)$, the thermal conductivity of the order (1-10) J per $\left(\mathrm{m} \mathrm{sec}{ }^{\circ} \mathrm{C}\right)$, and the specific heat of the order of $1000 \mathrm{~J}$ per $\left(\mathrm{kg}{ }^{\circ} \mathrm{C}\right)$, see e.g. Table 1 of [21,22]. Therefore the thermal diffusivity turns out to be $\left(10^{-6}-10^{-5}\right)\left(\mathrm{m}^{2} / \mathrm{s}\right)$. For example, for titanium $\alpha=9.0 \mathrm{x}$ $10^{-6}\left(\mathrm{~m}^{2} / \mathrm{s}\right)$ [34], while for ceramic $\alpha=6.2 \times 10^{-7}\left(\mathrm{~m}^{2} / \mathrm{s}\right)$ [35]. 


\section{APPENDIX B:}

We briefly summarize how to solve an ordinary, inhomogeneous first order linear differential equation. First, we find the solution of the corresponding homogeneous equation without the source term, and then we find a particular solution that satisfies the non-homogeneous equation.

So, if the equation has the form

$$
\dot{\mathrm{z}}+a z=\Gamma \exp (-\lambda t)
$$

with the initial condition $z(0)=z 0$, then the solution of the corresponding homogeneous equation is given by zo(t) $=\mathrm{C} \exp (-$ at) where $\mathrm{C}$ is an arbitrary constant. To obtain the partial solution we assume that it is of the same form with the source term, namely $z p(t)=\Delta \exp (-\lambda t)$, and by plugging it into the equation we find that $\Delta=\Gamma /(\alpha-\lambda)$. Taking the initial condition into account, the final expression for the solution is given by

$$
z(t)=\left(z_{0}+\frac{\Gamma}{\lambda-a}\right) \exp (-a t)+\frac{\Gamma}{a-\lambda} \exp (-\lambda t)
$$

To obtain the above solution it is assumed that the parameters a and $\lambda$ are different. However, in the special case in which they coincide, the partial solution of the differential equation

$$
\dot{\mathrm{z}}+a z=\Gamma \exp (-a t)
$$

turns out to be $z p(t)=\Gamma t \exp (-a t)$, and thus the full solution is given by

$$
z(t)=\left(z_{0}+\Gamma t\right) \exp (-a t)
$$




\section{References}

[1] Aida J, Ando $\mathrm{Y}$, Akhter R, Aoyama H, Masui M, Morita M. Reasons for permanent tooth extractions in Japan. J Epidemiol. 2006;16(5):214-9.

[2] Abnet CC, Qiao YL, Dawsey SM, Dong ZW, Taylor PR, Mark SD. Tooth loss is associated with increased risk of total death and death from upper gastrointestinal cancer, heart disease, and stroke in a Chinese population-based cohort. Int J Epidemiol. 2005;34:467-474.

[3] Holm-Pedersen P, Schultz-Larsen K, Christiansen N. Tooth Loss and Subsequent Disability and Mortality in Old Age. J Am Geriatr Soc. 2008;56:429-435.

[4] Holmlund A, Holm G, Lind L. Number of Teeth as a Predictor of Cardiovascular Mortality in a Cohort of 7,674 Subjects Followed for 12 Years. J Periodontol. 2010;81:870-876.

[5] Scheid RC, Woelfel JB. Woelfel's dental anatomy: Its relevance to Dentistry (ed 7). Baltimore, Lippincote Williams \& Wilkins, 2007.

[6] Marenzi G, Sammartino JC, Quaremba G, Graziano V, El Hassanin A, Qorri ME, Sammartino $\mathrm{G}$, Iorio-Siciliano V. Clinical Influence of Micromorphological Structure of Dental Implant Bone Drills. Biomed Res Int. 2018;2018:8143962.

[6a] Kalidindi V, "Optimization of drill design and coolant systems during dental implant surgery", University of Kentacky's Master's Theses, 2004.

[7] Linsuwanont $P$, Versluis A, Palamara JE, et al: Thermal stimulation causes tooth deformation: a possible alternative to the hydrodynamic theory? Arch Oral Biol 2008;53:261272.

[8] McCullagh P, Setchell DJ, Nesbit M, Biagioni PA, Lamey PJ. Infrared thermographic analysis of temperature rise on implant surfaces: a pilot study on abutment preparation. Pract Periodontics Aesthet Dent. 1998;10(9):1163-6, 1167.

[9] Gross M, Lanfer BZ, Ormianer Z. An investigation on heat transfer to the implant-bone interface due to abutment preparation with high-speed cutting instruments. Int J Oral Maxillofac Implants 1995;10(2):207-12.

[10] Bragger U., Wermuth W., Torok E: Heat generated during preparation of titanium implants of the ITI ${ }^{\circledR}$ Dental Implant System: an in vitro study. Clin Oral Implants Res 1995;6:254-9.

[11] Ormianer Z., Lanfer B-Z, Nissan J., Gross M: An Investigation of Heat Transfer to the Implant-Bone Interface Related to Exothermic Heat Generation during Setting of Autopolymerizing Acrylic Resins Applied Directly to an Implant Abutment. Int J Oral Maxillofac Implants 2000;15:837-42.

[12] Feuerstein O, Zeichner K, Imbari C, Ormianer Z, Samet N, Weiss El. Temperature changes in dental implants following exposure to hot substances in an ex vivo model. Clin. Oral Impl. Res. 19, 2008; 629-633.

[13] Nissan J., Gross M., Ormianer Z., Barnea E., Assif D., Heat Transfer of Impression Plasters to an Implant-Bone Interface. Implant Dent 2006;15:83-8. 
[14] Oskui IZ, Ashtiani MN, Hashemi A, et al: Effect of thermal stresses on the mechanism of tooth pain. J Endod 2014;40:1835-1839.

[15] Wood MR, Vermilyea SG, Committee on Research in Fixed Prosthodontics of the Academy of Fixed P. A review of selected dental literature on evidence-based treatment planning for dental implants: report of the Committee on Research in Fixed Prosthodontics of the Academy of Fixed Prosthodontics. J Prosthet Dent 2004;92(5):447-62.

[16] Li S, Chien S, Brånemark PI. Heat shock-induced necrosis and apoptosis in osteoblasts. J Orthop Res. 1999;17(6):891-9.

[17] Patel Z, Geerts GA. Temperature changes along a dental implant. Int J Prosthodont. 2011;24(1):58-63.

[18] Eriksson AR, Albrektsson T. Temperature threshold levels for heat-induced bone tissue injury: a vital-microscopic study in the rabbit. J Prosthet Dent. 1983;50(1):101-7.

[19] Eriksson RA, Albrektsson T. The effect of heat on bone regeneration: an experimental study in the rabbit using the bone growth chamber. J Oral Maxillofac Surg. 1984;42(11):705-11.

[20] Ormianer Z, Feuerstein O, Assad R, Samet N, Weiss El. In vivo changes in dental implant temperatures during hot beverage intake: a pilot study. Implant Dent. 2009;18(1):38-45.

[21] Palmer DS, Barco MT, Billy EJ. Temperature extremes produced orally by hot and cold liquids. J Prosthet Dent. 1992;67(3):325-7.

[22] Barclay CW, Spence D, Laird WR. Intra-oral temperatures during function. J Oral Rehabil. 2005;32(12):886-94.

[23] Wong K, Boyde A, Howell PG. A model of temperature transients in dental implants. Biomaterials. 2001;22(20):2795-7.

[24] Rabbani Arshad S, Zoljanahi Oskui I, Hashemi A. Thermal Analysis of Dental Implants in Mandibular Premolar Region: 3D FEM Study. J Prosthodont. 2018;27(3):284-289.

[25] Yeo IS, Lee JH, Kang TJ, Kim SK, Heo SJ, Koak JY, Park JM, Lee SY. The effect of abutment screw length on screw loosening in dental implants with external abutment connections after thermocycling. Int J Oral Maxillofac Implants. 2014;29(1):59-62.

[26] Çelik Köycü B, İmirzalıoğlu P. Heat Transfer and Thermal Stress Analysis of a Mandibular Molar Tooth Restored by Different Indirect Restorations Using a Three-Dimensional Finite Element Method. J Prosthodont. 2017;26(5):460-473.

[27] Oskui IZ, Ashtiani MN, Hashemi A, Jafarzadeh H. Thermal analysis of the intact mandibular premolar: a finite element analysis. Int Endod J. 2013;46(9):841-6.

[27a] Ma W., Liu W., Li M. Modeling heat transfer from warm water to foot: Analytical solution and experimental validation. International Journal of Thermal Sciences 98 (2015) 364-373.

[27b] Turkyilmazoglu M. Heat trasfer from warm water to a moving foot in a footbath. Applied Thermal Engineering 98 (2016) 280-287. 
[28] George B. Arfken, Hans J. Weber. Mathematical Methods for Physicists, 6th edition, Elsevier Academic Press, 2005.

[29] Spierings TA, Peters MC, Bosman F, Plasschaert AJ. Verification of theoretical modeling of heat transmission in teeth by in vivo experiments. J Dent Res. 1987;66(8):1336-9.

[30] Plant CG, Jones DW, Darvell BW. The heat evolved and temperatures attained during setting of restorative materials. Br Dent J. 1974;137(6):233-8.

[30a] Sapareto S.A. and Dewey W.C., Thermal dose determination in cancer therapy. Int J Radat Oncol Biol Phys, 10(6): p. 787-800 (1984)

[30b] https://www.ncbi.nlm.nih.gov/pmc/articles/PMC3799975

[31] de Vree JH, Spierings TA, Plasschaert AJ. A simulation model for transient thermal analysis of restored teeth. J Dent Res. 1983;62(6):756-9.

[32] Spierings TA, Peters MC, Bosman F, Plasschaert AJ. The influence of cavity geometry on heat transmission in restored teeth. J Dent. 1986;14(2):47-51.

[33] Fenner DN, Robinson PB, Cheung PM. Three-dimensional finite element analysis of thermal shock in a premolar with a composite resin MOD restoration.Med Eng Phys. 1998;20(4):269-75.

[34] Değer Y, Adigüzel Ö, Yiğit Özer S, Kaya S, Seyfioğlu Polat Z, Bozyel B. Evaluation of Temperature and Stress Distribution on 2 Different Post Systems Using 3-Dimensional Finite Element Analysis. Med Sci Monit. 2015;21:3716-172.

[35] Srivastava B, N Devi N, Gupta N, Singh R. Comparative Evaluation of Various Temperature Changes on Stress Distribution in Class II Mesial-occlusal-distal Preparation restored with Different Restorative Materials: A Finite Element Analysis. Int J Clin Pediatr Dent. 2018;11(3):167-170. 


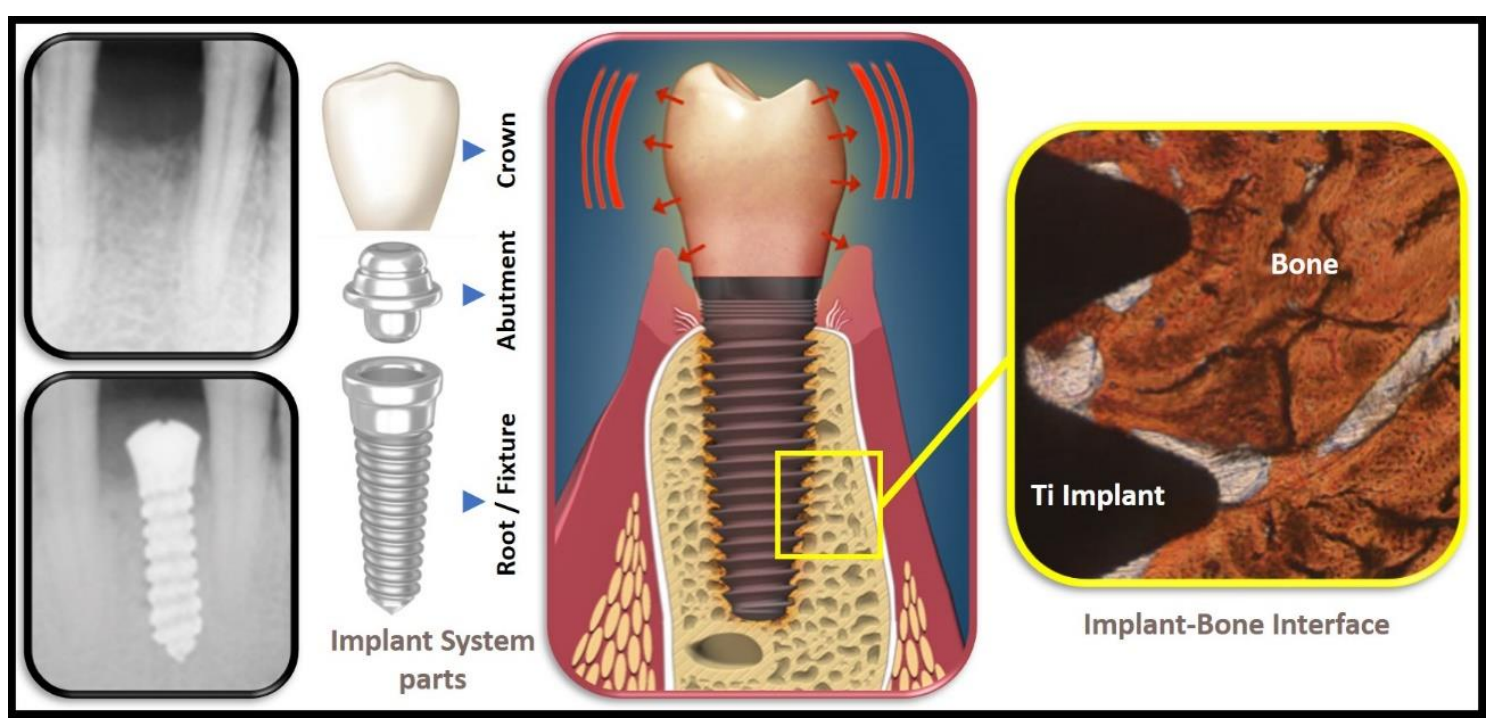

FIG. 1: Oro-Dental Implantology; illustrating main parts of a dental implant system and the implant-bone interface. 

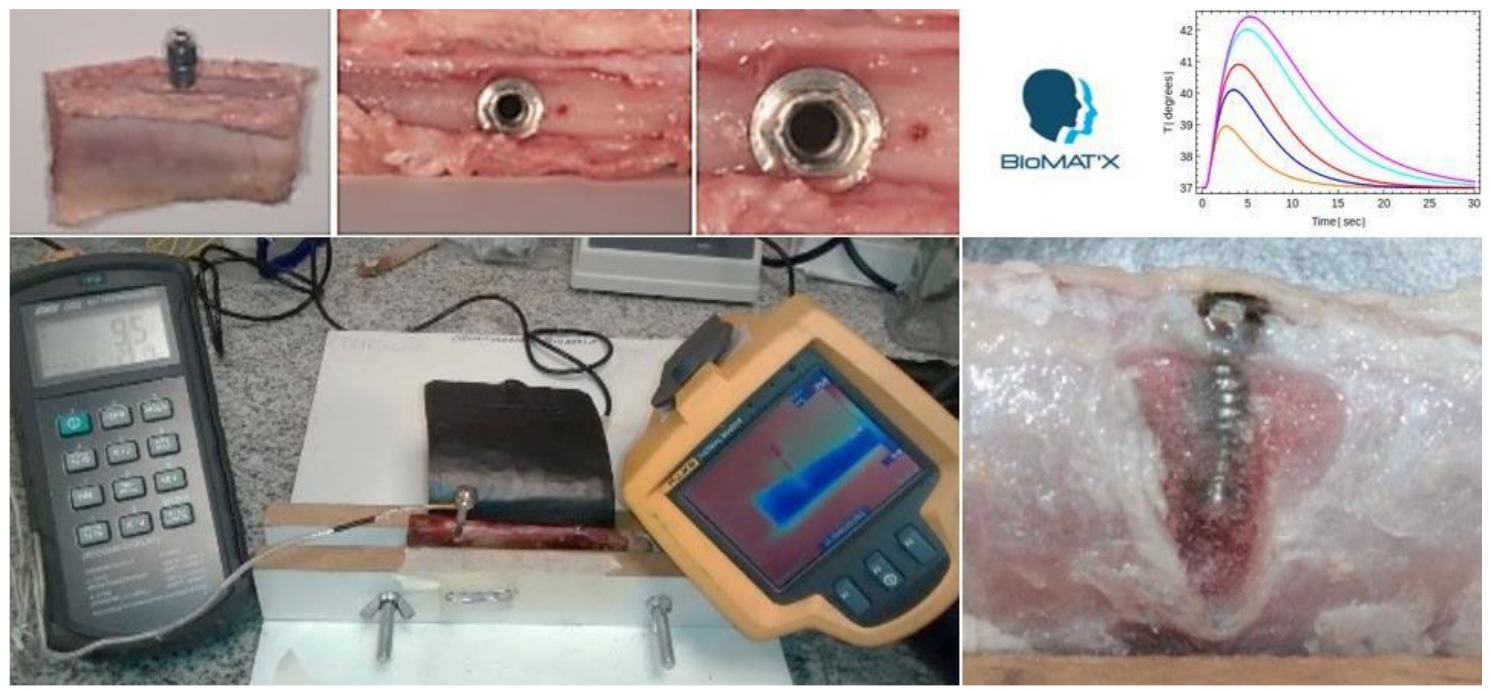

FIG. 2: Experimental Set-up; developed in-House as an ex-vivo heat distribution model employing human patient-grade titanium dental implants placed into porcine ribs (without coolant) and thermal changes monitored/recorded (quantified) using a CorDEX TP3R ToughPix DigiTherm Digital Thermal Camera. 


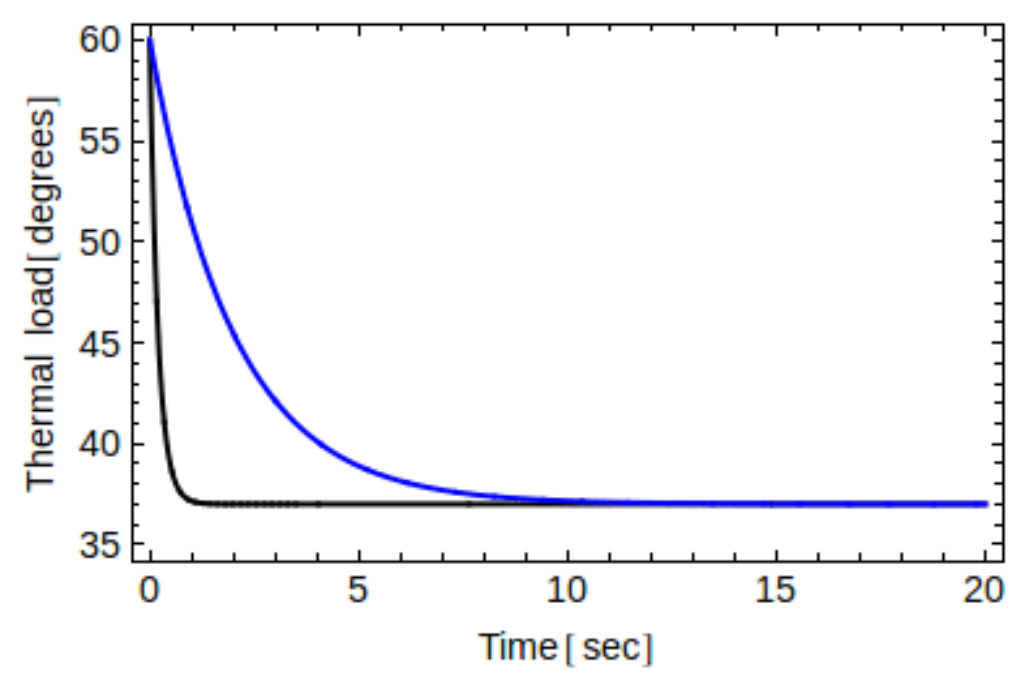

FIG. 3: Thermal load for two different exposure times, to $=0.2 \mathrm{sec}$ (black color) and to $=2 \mathrm{sec}$ (blue color). The first one drops faster. 


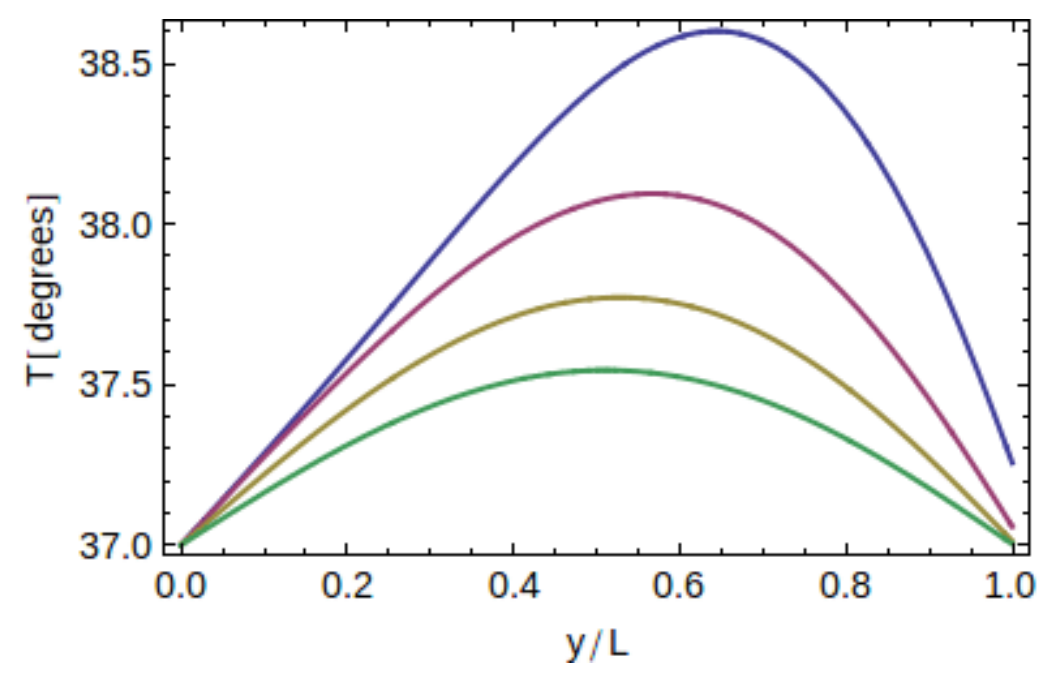

FIG. 4: Temperature distribution (in degrees ${ }^{\circ} \mathrm{C}$ ) versus location at four different instants of time (from top to bottom $9 \mathrm{sec}, 12 \mathrm{sec}, 15 \mathrm{sec}$ and $18 \mathrm{sec}$ ) for implant A () and for $\mathrm{t}_{0}=2 \mathrm{sec}$.

$\tau$

$\alpha=2 \times 10^{-6} \mathrm{~m}^{2} / \mathrm{sec}$ 


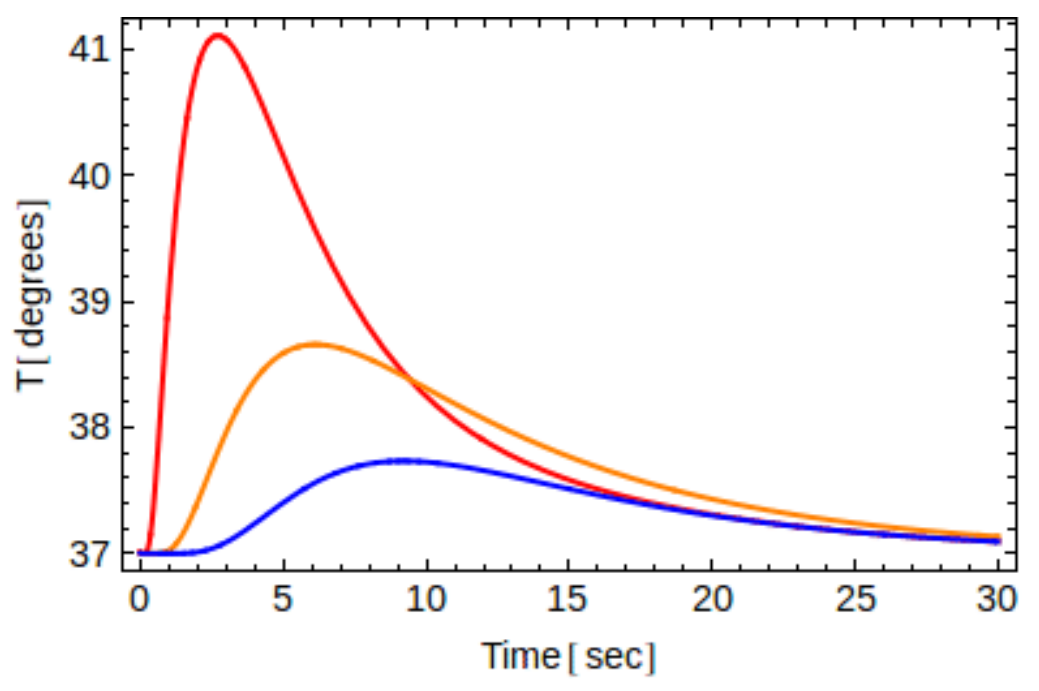

FIG. 5: Temperature changes (in degrees ${ }^{\circ} \mathrm{C}$ ) versus time (in sec) for implant $A$ and for $t_{0}=2 \mathrm{sec}$ at three different locations, namely $B 1(y=3 L / 4)$ in red, $B 2(y=L / 2)$ in orange, and $B 3(y=L / 4)$ in blue. 


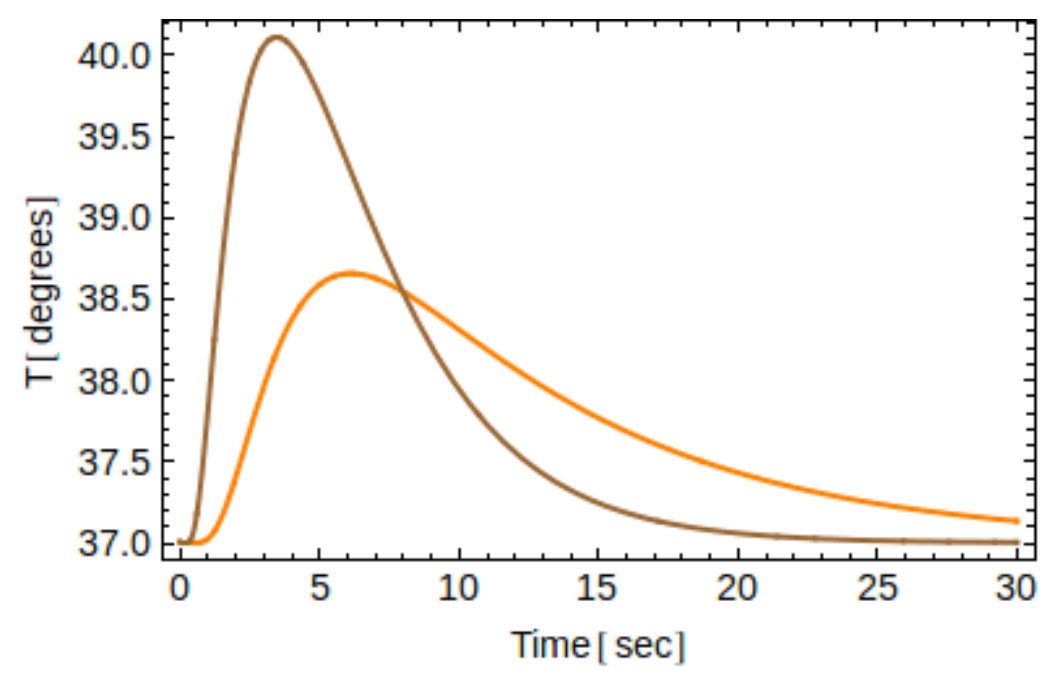

FIG. 6: Temperature changes versus time at point B2 for implants $A$ (orange) and B (brown) and for $t_{0}=2 \mathrm{sec}$. 


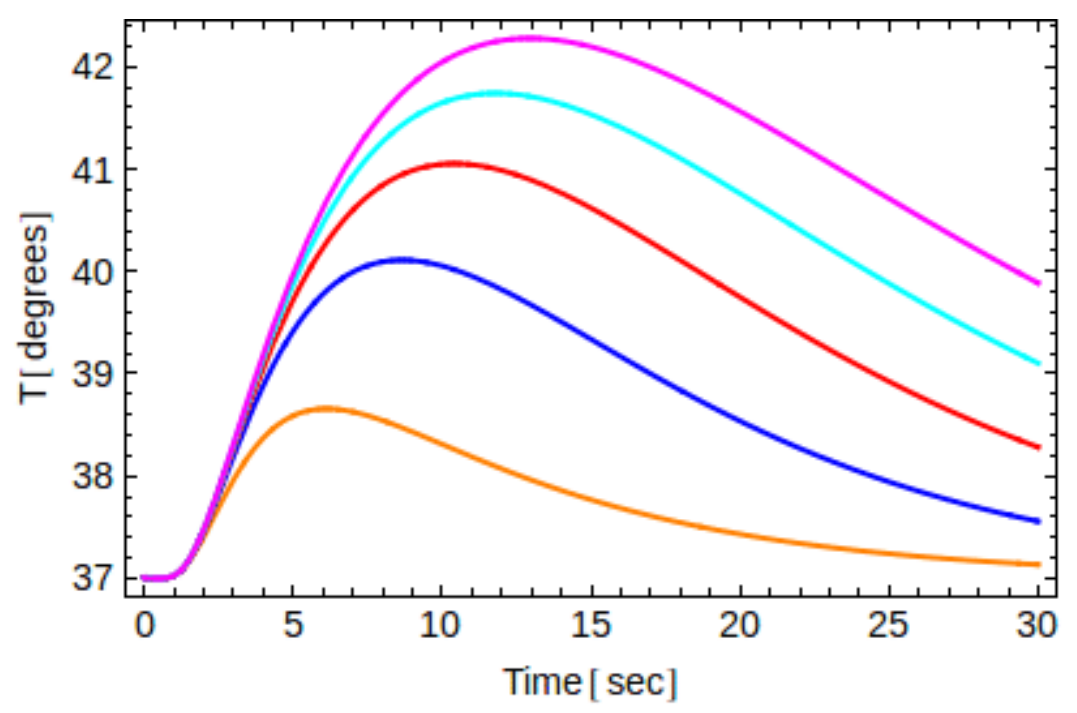

FIG. 7: Temperature changes versus time at point B2 for implant $A(=8.6 \mathrm{sec})$ and for $t_{0}=2 \mathrm{sec}$, $5 \mathrm{sec}, 8 \mathrm{sec}, 11 \mathrm{sec}$ and $14 \mathrm{sec}$ from bottom to top. 


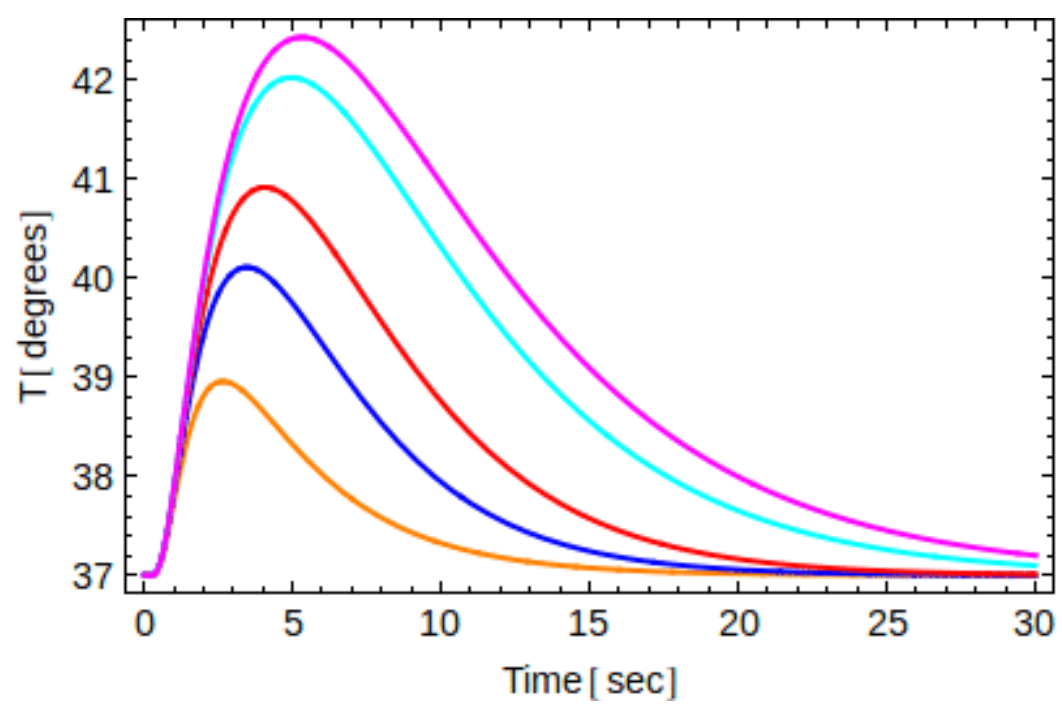

FIG. 8: Temperature changes versus time at point $B 2$ for implant $B(=3.4 \mathrm{sec})$ and for $t_{0}=1 \mathrm{sec}$, $2 \mathrm{sec}, 3 \mathrm{sec}, 5 \mathrm{sec}$ and $6 \mathrm{sec}$ from bottom to top. 